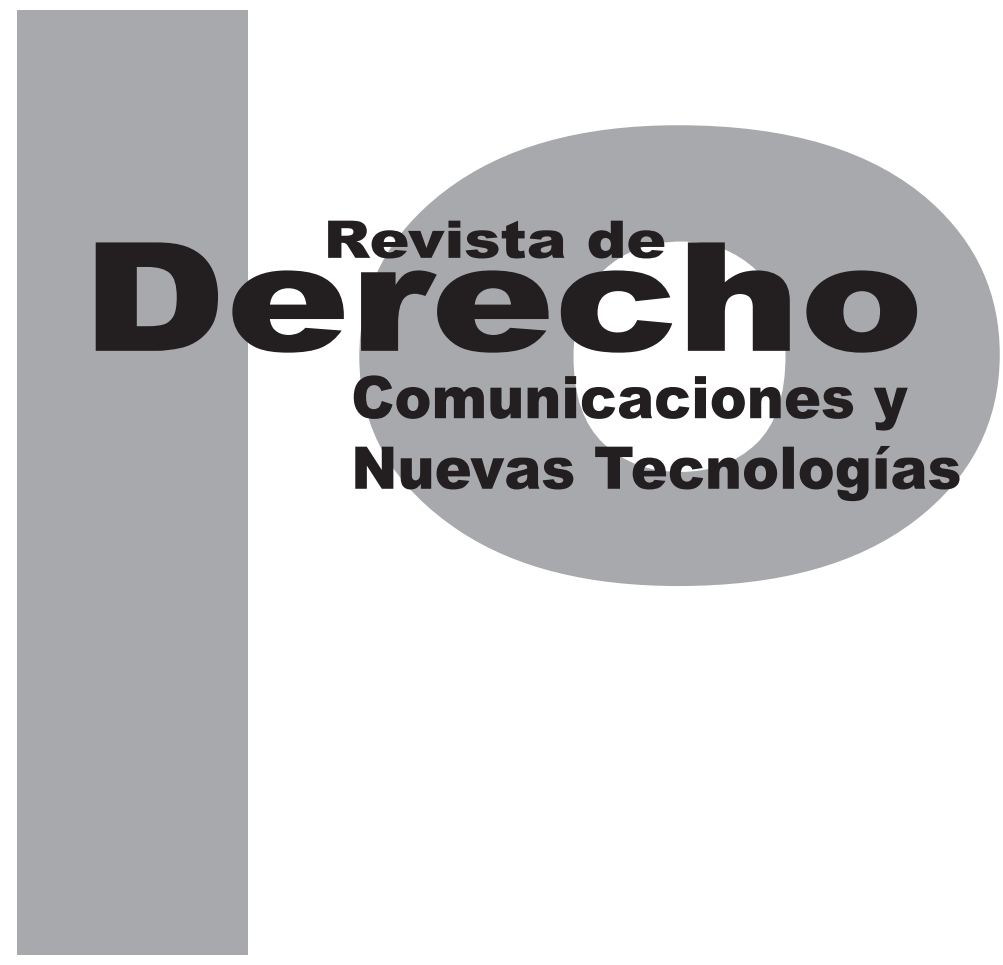

\title{
RÉGIMEN JURÍDICO DE LOS OBJETOS ESPACIALES
}

\author{
LAURA BOTERO URREA
}

Universidad de los Andes

Facultad de Derecho

Revista de Derecho, Comunicaciones y Nuevas Tecnologías

No. 10, Julio - Diciembre de 2013. ISSN 1909-7786 


\title{
Régimen jurídico de los objetos espaciales ${ }^{*}$
}

\author{
Laura Botero Urrea**
}

\section{RESUMEN}

El objetivo del presente trabajo es el análisis del Convenio sobre el registro de objetos lanzados al espacio ultraterrestre del año 1974, teniendo en cuenta la fundamentación para la creación del mismo y los deberes y obligaciones que éste trae para los Estados firmantes. Lo anterior, para concluir cuáles son las contribuciones que proporciona el convenio para la actividad espacial así como las fallas que posee. Adicionalmente se analiza el soporte que brindan las demás regulaciones conexas a éste.

Palabras clave: Objetos espaciales, Convenio de Registro, derecho espacial, actividad espacial, propiedad sobre objetos espaciales.

\section{ABStract}

The purpose of the following project is the analysis of the Convention on Registration of Objects Launched into Outer Space, taking into account the motives for its creation and the rights and obligations that the States are bound to follow. This will lead to identify its contribution to the outer space activity as well as its faults or mistakes. The additional contribution of the resolutions to this same subject is also analyzed.

KEYWORDS: Space objects, Registration Convention, Space Law, outer space activity.

Cómo citar este artículo: Botero Urrea, L. (Diciembre, 2013). Régimen jurídico de los objetos espaciales. Revista de Derecho, Comunicaciones y Nuevas Tecnologías, 10.

*** Abogada de la Universidad de los Andes en el año graduada en 2013. Actualmente ejerce como abogada en la empresa Avianca S.A. donde trabaja en el área de Asuntos Administrativos. Correos: I.botero34@uniandes.edu.co, laura.botero@avianca.com 


\section{SUMARIO}

Introducción -I. CLASIFICACIÓN DE OBJETOS ESPACIALES -A. Según su Carácter-B. Según su Recorrido -C. Según la presencia de Humanos II. CLASES DE OBJETOS ESPACIALES -A. Cohete Espacial -B. Lanzadera o Transbordador Espacial -C. Estaciones Espaciales -D. Satélites Artificiales -E. Sondas Espaciales -III RÉGIMEN JURÍDICO -A. Motivación del Tratado Sobre Registro de Objetos Lanzados al Espacio Ultraterrestre -B. Reglamentación Derivada del Tratado Sobre Registro de Objetos Lanzados al Espacio Ultraterrestre -C. Estudio de Resoluciones Conexas al Tratado -IV. BENEFICIOS EN LA APICACIÓN DEL TRATADO - A. Jurisdicción y Control -B. Aplicación de los Principios que deben regir las actividades espaciales -C. Aplicación efectiva de otros tratados - V. PROBLEMÁTICAS EN LA APLICACIÓN DEL TRATADO -A. Existencia de Otros Registros -B. Ratificación del Tratado -VI CONCLUSIÓN-Bibliografía 
Introducción

El Convenio sobre el registro de objetos lanzados al espacio ultraterrestre fue aprobado por la Asamblea General de la ONU en su resolución 3235 el día 12 de noviembre de 1974. Este convenio encuentra su fundamento en el Tratado sobre los principios que deben regir las actividades de los Estados en la exploración y utilización del espacio ultraterrestre, incluso la Luna y otros cuerpos celestes de 1967, en donde por primera vez se esboza la necesidad de un registro cuyo propósito sea otorgar la propiedad de objetos espaciales a los Estados, entre otras cosas.

Teniendo en cuenta que las relaciones de los Estados con el espacio ultraterrestre, la Luna y otros cuerpos celestes están limitadas por el principio de la no apropiación, bajo el cual ningún Estado podrá reclamar soberanía sobre él o las partes que lo componen ${ }^{1}$, la importancia del convenio bajo estudio radica en que éste representa a los objetos artificiales que sí son apropiables y que serán enviados al espacio bajo el entendido de que hacen parte de un Estado. Por lo anterior, los objetos artificiales espaciales son los únicos que aun llegando al espacio serán objeto de control y jurisdicción por parte de los Estados y no estarán sujetos al régimen de no apropiación de la Luna y otros cuerpos celestes, pues no se les puede catalogar como tales, sino como objetos hechos por el hombre, considerándose en el derecho como bienes muebles registrables como se explicará más adelante.

Según el Tratado sobre los principios que deben regir las actividades de los Estados en la exploración y Utilización del Espacio Ultraterrestre (1967).
Siendo esto así, resulta de vital importancia que sea clara y conocida por todos los Estados, la forma como se adquiere la jurisdicción y el control sobre un objeto cuya finalidad sea llegar al espacio; así como también debe ser de conocimiento universal qué Estados son propietarios de los objetos espaciales. Lo anterior tiene ciertas ventajas, como lo es la efectiva aplicación del Acuerdo sobre salvamento y devolución de astronautas y restitución de objetos espaciales y el Convenio sobre responsabilidad por objetos lanzados al espacio ultraterrestre, entre otras.

Teniendo en cuenta lo anterior, el objetivo principal de este artículo es el análisis del Convenio Sobre el Registro de Objetos Lanzados al Espacio, de 1974, el cual pretende enfocarse en las razones fundamentales por las cuales se realizó el tratado, las obligaciones y derechos que el mismo otorga, las ventajas de su aplicación así como las fallas del mismo y finalmente las posibles soluciones a estos problemas. Del mismo modo se analizarán los demás tratados y resoluciones que sirven de fundamento o complementan el convenio bajo examen.

La forma como se abordará el análisis anteriormente explicado es la siguiente: en primer lugar se definirá qué es un objeto espacial y se presentará la clasificación básica de los objetos espaciales, para que el lector comprenda cuál es el ámbito de aplicación del Tratado. Posteriormente, se llevará a cabo una lectura rigurosa y detenida de la parte motiva del tratado, es decir, del prefacio que describe las razones o propósitos que llevaron a convenir a los Estados sobre el registro de los objetos lanzados al espacio. 
De esta lectura se pretende extraer y explicar posteriormente la necesidad del tratado bajo estudio para la regulación de las relaciones de los países en el espacio ultraterrestre.

En segundo lugar, es pertinente revisar la mayoría, si no todos, los artículos del Tratado en aras de analizar qué es aquello que buscan reglamentar y bajo qué principios se fundamentan sus exigencias. Tendiendo esto claro, es posible analizar, posteriormente, las implicaciones (efectos positivos y negativos si los hay) de exigir un registro de estos objetos-bienes. Un ejemplo de ello sería la relación de éstos frente al régimen de responsabilidad de daños causados por objetos espaciales.

Finalmente es importante también realizar un análisis sobre las resoluciones que complementan el Tratado, puesto que pueden mejorar cuestiones que resultaban vagas inicialmente o complementar otras para hacerlo más específico. La importancia de estas resoluciones radica en que el convenio busca dar regulación al tema del registro de los objetos lanzados al espacio ultraterrestre y, por lo tanto, si hay más resoluciones que traten el tema, no es posible entender su regulación sin ellas.

De todo lo anterior se busca llegar a una conclusión sobre los beneficios que proporciona éste tratado en la práctica, así como aquellas cuestiones que podrían ser incluidas posteriormente si fuere el caso.

\section{CLASIFICACIÓN DE OBJETOS} ESPACIALES

Los objetos espaciales son bienes muebles registrables que tienen como finalidad llegar al espacio ultraterrestre. Son registrados como propiedad de uno de los Estados de lanzamiento, esto es, el Estado que lanza el objeto, que promueve el lanzamiento o desde cuyo territorio o instalaciones se lance el objeto. Para registrar los objetos espaciales existe la obligación de llevar un registro nacional por parte del Estado propietario y uno internacional, que es aquel que lleva el Secretario General de la ONU. Los objetos espaciales se componen tanto del vehículo propulsor como de la carga útil que éste pretende transportar.

Los objetos espaciales suelen clasificarse de distintas maneras y bajo criterios diferentes. Una de las formas de clasificación de los objetos espaciales más reconocida es aquella propuesta por el teórico argentino Manuel Augusto Ferrer, que se fundamenta en los siguientes criterios

\section{A. Según su carácter}

Pueden ser públicos o privados. Los primeros son aquellos que atienden a las necesidades de servicio de un Estado en particular. Los segundos se constituyen por objetos que cumplen una actividad o un propósito para agentes individuales desligados del poder estatal. 


\section{B. Según su recorrido}

Existen objetos orbitales y transespaciales. La clasificación de objetos orbitales se refiere a aquellos cuyo propósito es recorrer un camino alrededor de la tierra u otro cuerpo celeste ya sea de forma elíptica, hiperbólica o excéntrica. Este recorrido puede ser en una órbita fija, lo que implica que el objeto únicamente recorrerá una órbita determinada según lo programa el responsable del lanzamiento, o, una órbita variable que indica que el objeto espacial cambiará su órbita ya sea porque así esta previsto por el Estado responsable o porque de forma natural (si no es un objeto artificial) la cambia.

Por otro lado, los objetos espaciales que realizan recorridos transespaciales son aquellos diseñados para desplazarse de un cuerpo celeste a otro, por ejemplo, de la tierra a la Luna o a Marte.

\section{Según la presencia de humanos}

Los objetos pueden ser tripulados o no tripulados. Aquellos tripulados son los diseñados para llevar personal humano al espacio y lo no tripulados son aquellos manejados por el hombre desde tierra dentro de los cuales no hay cabida para la presencia de personal humano. (Ferrer, 1976, Derecho Espacial, p. 250-260).

\section{CLASES DE OBJETOS ESPACIALES}

Teniendo en cuenta los criterios anteriores, es importante mencionar que existen dos clases de objetos espaciales las cuales son el vehícu- lo propulsor y la carga útil. Estas a su vez se dividen en diferentes tipos ya que existen diferentes características dentro de la misma clasificación que corresponden a diversos diseños y propósitos.

\section{A. Cohete espacial}

Por el término propulsión espacial se entiende cualquier tecnología que tenga la capacidad de proporcionar impulso a un objeto hacia el espacio ultraterrestre y alrededor de éste. (Asamblea general de las Naciones Unidas, 1971, Convenio sobre la responsabilidad internacional por daños causados por objetos espaciales, Art. 1, lit. d). Teniendo en cuenta esta definición, un cohete espacial puede ser considerado como un vehículo propulsor. (Duque, y Pérez, 2012, Objetos Espaciales. Derecho del Espacio Ultraterrestre, p. 13).

El cohete espacial es un objeto que contiene un motor de propulsión que funciona por medio de la combustión de gases. La combustión genera suficiente energía cinética la cual se canaliza a través de un tubo de propulsión permitiendo al objeto romper con la fuerza de atracción (entendida como gravedad), logrando impulsarlo al espacio ultraterrestre. Este tipo de vehículo propulsor es utilizado tanto para llevar carga útil como satélites o personal humano.

Existen además distintas clases de cohetes según el tipo de combustible que se utiliza y según las fases que contiene. En cuanto al tipo de combustible, un cohete puede utilizar combustible líquido, para lo cual el propelente y el oxi- 
dante se encuentran separados y se mezclan en la cámara de combustión. También puede utilizar combustible sólido, lo que implica que tanto el propelente como el oxidante se encuentran mezclados en la cámara de combustión en un estado sólido.

Refiriéndose a las fases, los cohetes pueden tener una sola fase o múltiples fases. Aquellos que solo poseen una, realizan el recorrido desde la tierra hasta su lugar predeterminado en el espacio sin dividirse, ya que el combustible que requiere esta contenido en una sola cámara. De forma contraria, el cohete con múltiples fases se divide durante su recorrido según cada fase vaya agotando el combustible. Esto permite que el cohete tenga más capacidad ya que puede abarcar mucho más combustible. No obstante, esto también implica una mayor contaminación espacial.

\section{B. Lanzadera espacial o transbordador espacial}

Se puede afirmar que este objeto es una especie de mezcla entre un avión y un cohete, ya que su forma de despegue corresponde a la misma de los cohetes, con la diferencia de que debido a su estructura de avión tiene la posibilidad de volver a la tierra, lo que le permite realizar diversos viajes. En su estructura, consta de un orbitador, que es en efecto la nave que pretende desplazarse en el espacio ultraterrestre. También cuenta con aceleradores, que son los cohetes que propulsan el orbitador fuera de la atmósfera terrestre.
C. Estaciones Espaciales

Las estaciones espaciales son objetos diseñados para realizar misiones y actividades en el espacio ultraterrestre que orbitan alrededor de la tierra o de cualquier otro cuerpo celeste según la intención de la misma. Estas no contienen un sistema de propulsión como los objetos anteriormente expuestos, por lo que su construcción depende de que otros vehículos propulsores lleven sus partes para ser conectadas en el espacio. Es por esto que las estaciones espaciales están compuestas de módulos, cada uno con diferentes características y usos. Asimismo cuentan con una clase de objetos denominadas brazos robóticos que se desplazan sobre las estaciones espaciales con el propósito de repararlas.

Un ejemplo actual es la Estación Espacial Internacional, que cuenta con astronautas de diversas nacionalidades unidos por el propósito de realizar contribuciones científicas. Se puede afirmar que la Estación Espacial Internacional es un laboratorio en el espacio ultraterrestre.

\section{Satélites Artificiales}

La palabra satélite es utilizada actualmente de forma indiscriminada para denominar aquellos objetos que el hombre lanza al espacio ultraterrestre y que orbitan la tierra con distintos fines. No obstante es importante hacer hincapié en que el término correcto para denominar lo anterior es el de satélite artificial. Esto se fundamenta en que la palabra satélite se emplea en el derecho espacial para aquellos cuerpos naturales que orbitan alrededor de cuerpos ce- 
lestes, como por ejemplo las lunas que orbitan alrededor de Saturno.

Los satélites artificiales pueden recorrer distintas órbitas alrededor de la tierra según la actividad que realicen, . por ejemplo, la órbita baja o LEO, por sus siglas en inglés, que debido a su cercanía con la tierra es la más utilizada por el bajo costo que implica ubicar los satélites en ella. También existe la órbita mediana o MEO, por sus siglas en inglés, que se utiliza generalmente para comunicación de telefonía y televisión. La órbita de los satélites geoestacionarios - GEO es la más apetecida por los Estados, pues en esta los satélites giran a la misma velocidad de la tierra. Finalmente la órbita alta o HEO, por sus siglas en inglés, es la más alejada de la tierra, razón por la cual se ha destinado para enviar a los satélites que estaban en la GEO que han caído en desuso.

\section{E. Sondas Espaciales}

Las sondas espaciales son objetos enviados al espacio con el propósito de realizar estudios sobre cuerpos celestes. Estos estudios pueden realizarse por medio del aterrizaje de la sonda en el cuerpo celeste o simplemente orbitando al rededor del mismo, debido a que las sondas no son objetos tripulados, son manejadas por el hombre desde la tierra.

\section{RÉGIMEN JURÍDICO}

\section{A. Motivación del Tratado Sobre Registro de Objetos Lanzados al Espacio Ultraterrestre}

La parte motiva del Tratado mencionado resulta de gran importancia cuando se quiere buscar la intención y propósitos que pretende alcanzar la existencia del mismo, así como las necesidades que éste aspira satisfacer en referencia a problemas que pudieron presentarse en el momento frente a la actividad espacial. Es importante conocer y entender las motivaciones de un tratado, es decir, las razones que dieron lugar a su conformación, para que éste sea interpretado con base a tales razones ya que una interpretación alejada de las mismas desnaturalizaría su propósito. En este sentido, se realizará a continuación el análisis de cada uno de los puntos que son relevantes para comprender el tratado.

El tratado, comienza "Reconociendo el interés común de toda la humanidad en proseguir la exploración y utilización del espacio ultraterrestre con fines pacíficos" (Asamblea Gerneral de las Naciones Unidas, 1974, Convenio sobre el registro de objetos lanzados al espacio ultraterrestre). Esta declaración es de inmensa importancia si se tiene en cuenta que desde los inicios de la actividad espacial los países han enfocado todos sus esfuerzos en mantener el espacio ultraterrestre libre de los peligros de la guerra, pues los efectos de que se desatara un conflicto armado en el espacio ultraterrestre presentan un riesgo mayor. En razón de esto, 
para los Estados Parte es menester reconocer que cada tratado o resolución que se convenga es siempre con el fin de mantener el uso pacífico del espacio ultraterrestre.

En segundo lugar, se hace mención al Tratado sobre los principios que deben regir las actividades de los Estados en la exploración y utilización del espacio ultraterrestre incluso la luna y otros cuerpos celestes, de 1967. Así pues, este tratado es uno de los fundamentos del convenio sub examine, pues es en donde por primera vez se establece que el Estado que registre un objeto como propio mantendrá la jurisdicción y control sobre el mismo. (Asamblea General de las Naciones Unidas. (1967). Tratado sobre los principios que deben regir las actividades de los Estados en la exploración y utilización del espacio ultraterrestre, incluso la Luna y otros cuerpos celestes, Art. 8).

Siguiendo el tratado se observa que se hace mención al Acuerdo sobre el salvamento y devolución de astronautas y la restitución de objetos lanzados al espacio ultraterrestre, del 22 de abril de 1968. Este acuerdo establece, entre otras cosas, ciertas pautas a seguir cuando un Estado encuentre en su territorio un objeto espacial que no es de su propiedad. Dentro de éstas se encuentra la notificación de tal evento a la autoridad de lanzamiento y la devolución al mismo. El mandato anterior encuentra muchas dificultades si el objeto que se pretende restituir no esta identificado como propiedad de un Estado, pues no es posible saber a quién se devuelve. Es por eso que el Convenio bajo estudio mejora la situación anterior en dos aspectos importantes: por una parte, facilita la identificación de un objeto "perdido" por parte del Estado bajo cuyo territorio se encuentre y obliga a éste a restituirlo. Por otra parte, otorga el derecho al Estado propietario a recuperarlo.

Más adelante se hace alusión al Convenio sobre la responsabilidad internacional por daños causados por objetos espaciales, del 29 de marzo de 1972, el cual establece los procedimientos y normas relativos a la responsabilidad de los Estados de lanzamiento frente a daños ocasionados por un objeto de su propiedad. Como ya se había mencionado es necesario conocer quién es el propietario para saber en cabeza de quién está la responsabilidad por un daño. Por lo tanto, sin la existencia del Tratado bajo estudio, el convenio de responsabilidad no encuentra aplicación alguna, puesto que sin el registro es imposible saber quién es el propietario, cuál es el Estado de lanzamiento y así, de quién es la responsabilidad por el daño causado por el objeto espacial.

Nuevamente se hace referencia al Tratado sobre los principios que deben regir las actividades de los estados en la exploración y utilización del espacio ultraterrestre incluso la Luna y otros cuerpos celestes, pero esta vez para expresar que el Tratado solicita que se establezcan disposiciones referentes al registro nacional por parte de los Estados de lanzamiento. Por lo tanto, esta disposición se desarrolla en el Convenio y pretende que la actividad ultraterrestre pueda llevarse a cabo según los principios establecidos en el Tratado del 67. La importancia de un registro nacional por parte de los países que 
lancen un objeto es que la información que aquí se establezca debe ser presentada a la ONU por medio del Secretario General, para que ésta, con base en tal registro, pueda hacer su propio registro.

Avanza el tratado en la concreción que se deriva de las razones expuestas, en el deseo de establecer las normas pertinentes para crear un registro obligatorio que sea llevado por el Secretario General de la ONU. Es importante hacer hincapié en la palabra "obligatorio", ya que deja a un lado la discrecionalidad e impone que todo lo que se va a reglamentar a continuación es un requisito ineludible para todos los Estados parte del tratado si se quiere enviar un objeto al espacio. Por lo anterior, es necesario establecer de forma clara y expresa, para que sea de conocimiento de todos los Estados, qué debe contener tal registro para que éste sea único e inconfundible para todos los Estados de lanzamiento.

Resulta claro que este punto refleja el fin principal del Tratado en cuestión, el de establecer los procedimientos, normas, requisitos etc., referentes a la realización del registro, determinar la obligación que tienen los Estados de lanzamiento con cuestiones relacionadas al mismo (como es deber de informar del lanzamiento y el deber de ayudar en la identificación de objetos que salgan del lugar de conocimiento del Estado propietario), así como también los derechos multilaterales que nacen para todos los Estados por la creación del mismo, como lo es la libertad de acceso a los registros de los otros Estados. Todo lo anterior busca satisfacer aquellas cuestiones que ya se describieron en los párrafos anteriores.
Finalmente, se expresa que el registro, materia de desarrollo en el presente Convenio, proveerá a los Estados de lanzamiento ayuda en cuanto a la identificación de sus propios objetos y los de los demás Estados de lanzamiento. Además se expresa que contribuirá a la aplicación del Derecho Internacional que regula la actividad en el espacio ultraterrestre, puesto que ayudará a que la aplicación de los otros tratados mejore y se amplíe, como lo es específicamente en el caso del tratado de 1967 en el Acuerdo sobre el salvamento y devolución de astronautas y la restitución de objetos lanzados al espacio ultraterrestre, del 22 de abril de 1968, y en el Convenio sobre la responsabilidad internacional por daños causados por objetos espaciales, del 29 de noviembre de 1971.

\section{B. Reglamentación Derivada del Tratado Sobre Registro de Objetos Lanzados al Espacio Ultraterrestre}

Analizada la motivación del tratado sub examine y bajo el entendido que éste debe interpretarse y aplicarse según los motivos que le dan origen, se pasará al estudio detallado de cada uno de los artículos que contiene, pues son todos estos de cumplimiento obligatorio para todos los Estados que pretendan lanzar un objeto al espacio ultraterrestre y de su acertado entendimiento, radica que se apliquen de forma adecuada.

Define el artículo I del Tratado bajo estudio el concepto de "estado de lanzamiento", el cual es de vital importancia para que los Estados tengan conocimiento sobre quién puede llegar a ser un Estado de lanzamiento según su forma de parti- 
cipación en el lanzamiento de un objeto al espacio ultraterrestre. Es importante poder hacer tal identificación de forma correcta, ya que esta situación derivará para el o los Estados que se inscriban como tal ciertas obligaciones y derechos.

Así pues, por "estado de lanzamiento" se debe entender:

1.) El Estado que lance un objeto espacial. Lo anterior debe entenderse de forma restrictiva, pues abarca únicamente la actividad de lanzar, efectivamente, un objeto espacial.

2.) Un Estado desde cuyo territorio se lance un objeto espacial. Esta concepción no requiere mayor explicación, pues se refiere al territorio que le pertenece a determinado Estado y desde el cual se realiza el lanzamiento.

3.) El Estado desde cuyas instalaciones se lance un objeto espacial. Para comprender este criterio es importante saber que debido a las diferencias en materia de desarrollo espacial de los Estados, en la práctica sucede que algunos de ellos prestan sus instalaciones para que otros realicen sus lanzamientos aun cuando éstos no vayan a ejercer la jurisdicción y el control sobre el objeto. En otros eventos, existen también organizaciones internacionales espaciales que tienen sus instalaciones en otros territorios. Por lo tanto, un Estado u organización que lance un objeto desde sus instalaciones, cuando estas se encuentran en un territorio distinto a su jurisdicción, también será identificado como un Estado de lanzamiento.
Como es evidente, los criterios expuestos no presentan mayor problema en su identificación, pero el siguiente puede resultar muy amplio ya que tiende a tener distintas interpretaciones.

4.) El estado que promueva el lanzamiento de un objeto espacial. Para comprender este criterio es menester tener en cuenta la relación entre el Estado y el objeto espacial, dejando a un lado quién realizó la actividad estricta de lanzarlo. Por lo tanto, el Estado al cual se le atribuye tal relación es aquel sin cuya autorización explícita o contribución, el objeto nunca hubiera alcanzado el espacio ultraterrestre. (Benko, \& Schrogl, 2005, Essential Air and Space Law, p. 132-133). Por lo anterior y si se acepta este tipo de interpretación, se pueden incluir dentro del criterio mencionado las siguientes situaciones:

A. Objetos espaciales lanzados con el propósito de cumplir servicios públicos ya sea de manera parcial o total. En este ejemplo no es relevante si el Estado utiliza o no sus propias instalaciones o si en efecto financia el lanzamiento, pues el hecho de que el objeto espacial vaya a ser utilizado con fines públicos, ya compromete al Estado en relación con el objeto.

B. Objetos espaciales lanzados con la finalidad de cumplir intereses o servicios privados o comerciales, parcialmente financiados por el sector público. En esta situación, aun cuando la actividad tenga intereses del sector privado, la intervención financiera 
del Estado hace que se consolide necesariamente una relación entre éste y el objeto espacial.

C. Objetos lanzados para cumplir actividades puramente privadas de entidades no gubernamentales. En este ejemplo final se entiende que a pesar de que la actividad sea meramente privada, la organización debe estar circunscrita a las leyes de algún Estado y así, éste se ve relacionado con el objeto espacial lanzado.

Se puede deducir de los anteriores significados que el concepto de "Estado de lanzamiento", busca involucrar a todos los Estados que tengan de uno u otro modo participación en un lanzamiento, para no limitar responsabilidades de forma injusta ya que las acciones de prestar el territorio, promover o efectivamente lanzar el objeto, son todas acciones constitutivas de responsabilidades.

Es menester hacer hincapié sobre el hecho de que anterior a la definición sobre el concepto descrito, existen dos tratados que se refieren a él. Estos son los Tratados sobre responsabilidad por objetos lanzados al espacio ultraterrestre y el Convenio sobre la devolución de astronautas y restitución de objetos lanzados al espacio. Frente al primero no existe ninguna problemática pues el concepto se describe de la misma forma como se realiza en este Tratado. No obstante, frente al segundo se encuentra una diferencia que en un principio puede parecer problemática, pero que se resuelve de manera sencilla cuando se comprenden ambos conceptos de manera armónica.
Es así como se encuentra que en el artículo VI del Acuerdo sobre salvamento y devolución de astronautas y restitución de objetos lanzados al espacio ultraterrestre se establece que: "se entenderá por autoridad de lanzamiento', el estado responsable del lanzamiento". Como se evidencia, este Convenio no habla de "Estado de lanzamiento sino de "autoridad de lanzamiento" y limita esta definición a un Estado u organización internacional que lance un objeto espacial. Con respecto a esto se puede decir que debido a que esta definición pertenece a un Tratado anterior al sub examine y anterior al Tratado de responsabilidad, tal vez no se habían previsto para esa época los diversos modos de participación que pueden existir entre los Estados al lanzar un objeto espacial y que sí fueron previstas por los tratados posteriores. Por esta razón, debe comprenderse que el concepto de "autoridad de lanzamiento" es equiparable al de "Estado de lanzamiento" y como tal, comprende todas aquellas posibilidades de participación en las que puede incurrir un Estado en un lanzamiento como lo son, la promoción, el territorio, las instalaciones y el lanzamiento en sentido estricto, y no únicamente la descrita por él.

Seguidamente, es necesario comprender qué es un “objeto espacial” para efectos del Tratado y para efectos del Derecho Internacional espacial, en aras de saber a qué se le aplica el presente Tratado. Según el artículo VI, un objeto espacial comprende dos partes que conforman conjuntamente la definición de "objeto espacial". Estas partes corresponden a: 1. El objeto que se pretende enviar al espacio para cumplir ciertas funciones, es decir, la carga útil, 
por ejemplo, el satélite de telecomunicaciones o de teleobservación de la tierra que cierto país pretende posicionar en el espacio, y 2. El vehículo que transporta la carga útil al espacio, por ejemplo, el cohete que va a trasladar el satélite. De esta definición se puede concluir que todo objeto que tenga como fin cumplir una función en el espacio ultraterrestre, ya sea como medio de transporte desde tierra o para el funcionamiento posterior en el espacio, se considera un objeto espacial.

Finalmente, el artículo I expone un tercer concepto, el cual es el de "Estado de registro". El "Estado de registro" es entonces el Estado que figura como propietario del objeto espacial frente a la comunidad internacional, es decir, aquel que ostenta la jurisdicción y el control del objeto que orbita. De cierta forma, es este concepto el que le da la importancia al Tratado bajo análisis, pues de su correcto entendimiento se deriva la efectiva aplicación del mismo, como también la razón de expedición, la cual es otorgar la propiedad a los objetos artificiales que pretendan ser enviados al espacio ultraterrestre.

Trae luego el Tratado, en el artículo II, tres puntos importantes para los cuales es importante tener en cuenta el concepto sobre "Estado de registro" ya expuesto. El primero dispone que el Estado de lanzamiento debe registrar el satélite (es decir, inscribir su propiedad) por medio de la inscripción de éste en un registro que él deberá llevar (registro nacional) y, además, del cual debe notificar al Secretario General de la ONU.
Si se tiene en cuenta la definición de Estado de lanzamiento, se sabe que este no es siempre un Estado por sí solo, sino que frente a un objeto espacial pueden existir varios Estados de lanzamiento según la forma en que cada uno haya participado. Para tales efectos, el artículo bajo estudio dispone en el punto dos, que los Estados de lanzamiento deben ponerse de acuerdo sobre cuál de ellos registrará el objeto. Explica también que independientemente del Estado que registre un objeto espacial como propio, los Estados de lanzamiento pueden ponerse de acuerdo sobre quién efectivamente va a ejercer el control sobre el mismo. No obstante, este punto hace énfasis en que el artículo debe entenderse en armonía con el artículo VIII del Tratado de 1967 en donde se establece que, para efectos internacionales y frente a terceros, se entiende que el Estado de registro es el propietario del objeto espacial, aun cuando entre los varios Estados de lanzamiento se haya acordado otra cosa. No quiere esto decir que los acuerdos entre los Estados de lanzamiento frente a la propiedad, control, etc. del objeto espacial sean inválidos, sino que para efectos de practicidad, los terceros entienden que el propietario es el Estado que lo haya registrado.

Finalmente, en un tercer punto, el artículo establece que la forma como se lleve el registro nacional y el contenido del mismo es discrecionalidad del Estado de registro. Más adelante se mostrará como este artículo supone un problema ya que la forma como cada Estado nombra las características (el periodo nodal, la inclinación, el perigeo, las unidades de medición y tiempo, etc.) se presta para confusiones. No obstante, 
se han realizado avances frente a este problema que serán discutidos con posterioridad. Es importante tener en cuenta que el registro al que se esta haciendo mención, es el registro nacional que lleva cada Estado.

Como complemento del artículo anterior, el artículo III establece que además del registro nacional, toda la información en éste debe notificársele también al Secretario General de la ONU, en aras de que existan también en el orden internacional los registros de cada objeto que se lanza al espacio ultraterrestre.

Establece también que tanto este registro como la información que contiene es de libre acceso para todos los Estados, sin restricción alguna. Esto es importante ya que materializa el principio de reciprocidad en cuanto a que todos los Estados tienen la posibilidad de saber qué actividades espaciales están realizando la totalidad de los Estados y con qué vehículos u objetos lo hacen.

Para dar aplicación al artículo III, el artículo IV especifica qué debe contener el registro internacional:

A) el nombre o nombres de los Estados de lanzamiento. Aunque sea solo un Estado de lanzamiento quien hace la inscripción del objeto espacial en su registro, es necesario que se ponga en conocimiento del Secretario General de la ONU, cuales son los estados que colaboraron e incidieron en el lanzamiento del objeto. B) Un nombre o número de registro bajo el cual se pueda identificar el objeto espacial. Esto sirve para corroborar a quién pertenece un objeto espacial o parte de éste que se haya perdido, pues como se explicó al principio el estado quien lo encuentre puede pedir información legal que demuestre que quien lo reclama es realmente el propietario. C) Fecha en que se lanzará el objeto y territorio desde donde se pretende lanzar. D) Información básica del objeto con respecto a la órbita que recorre: periodo nodal, (número de veces que pasa el objeto espacial por el mismo punto en determinado tiempo), inclinación, (ángulo de inclinación del objeto con respecto a la línea ecuatorial), Apogeo, (punto máximo de altura al cual llega el satélite, es decir, el punto más alejado de la tierra que alcanza el satélite en una órbita excéntrica), y perigeo, (la distancia más cercana de la tierra que alcanza el satélite en igual clase de órbita). E) Función general del satélite, es decir con qué propósito se lanzó, por ejemplo para cumplir funciones meteorológicas, de telecomunicaciones, GPS, etc. (Asamblea General de las Naciones Unidas, 1974, Art. 4).

Accesoriamente, el artículo IV dispone que cualquier arreglo o información adicional que resulte debe ser registrada, así como también si el satélite ha salido de funcionamiento. Este requerimiento ese da en aras de mantener los registros actualizados y así dar a la institución y a los países un conocimiento acertado sobre lo que esta ocurriendo constantemente en el espacio ultraterrestre. Asimismo y en cuanto a la órbita geoestacionaria se refiere, este requerimiento permite la aplicación del uso eficaz y equitativo ${ }^{2}$ de la misma, pues permite identificar cuándo hay una posición orbital disponible para ser utilizada por otros Estados.

2 Algunos aspectos relativos a la utilización de la órbita de los satélites geoestacionarios. 
Atendiendo al registro de información adicional que plasma el artículo anterior, el artículo $\mathrm{V}$ establece que el Estado de registro debe informar al Secretario General si un objeto espacial registrado a su nombre con la identificación que impone el artículo IV, literal B, ha sido lanzado. Esto en aras de tener conocimiento sobre si se dio efectivamente el lanzamiento que estaba programado para la fecha registrada o no. Hay una relevancia importante frente al tema, ya que de no lanzar el objeto en la fecha programada, comienza a correr un término dentro del cual el Estado debe lanzarlo so pena de perder la posición orbital asignada. De igual forma, lo anterior da aplicación a los principios que rigen a los satélites de la órbita geoestacionaria, ya que si se pierde una posición orbital por parte de un país esta puede asignársele a otro.

Más adelante, en el artículo VI, se hace referencia a la obligación que tienen todos los Estados de ayudar en la identificación de un objeto espacial que haya causado algún daño a otro. Esta obligación se deriva del principio de cooperación y encuentra su fundamento en que si un Estado fue receptor de un daño, no se le puede imponer una carga adicional que sea la de identificar por sí solo el objeto que lo causó. Esto implica que si algún Estado posee información sobre el daño que causó un objeto espacial a otro, debe suministrarla para su identificación. Esto permite agilidad en la búsqueda del responsable y así una indemnización más rápida por el daño causado. Para que el Estado a quien se le causó el daño reciba la ayuda por parte de los demás Estados, debe hacer una solicitud de forma autónoma o por medio del Secretario
General de la ONU en su nombre, presentando toda la información que tenga en su poder que resulte relevante para la identificación del Estado que ocasionó el daño.

El artículo VII trae a colación un asunto importante, ya que logra prever situaciones que a futuro serían más comunes que lo que eran en ese momento. Esto es, la aceptación de que las organizaciones intergubernamentales internacionales también pueden realizar actividades espaciales en nombre propio y que por lo tanto, en virtud de este Convenio, pueden obtener beneficios como también obligaciones. Esto esta sujeto claramente a la firma del Convenio por parte de la organización o que la mayoría de los Estados que la integran hagan parte del mismo. El Tratado dice específicamente que en todos los artículos en que se hace referencia a Ios "Estados", debe entenderse esta expresión de manera amplia, de forma tal que las organizaciones intergubernamentales internacionales estén contenidas dentro de la expresión. Los únicos artículos que excepcionan esta regla son Ios artículos VIII a XII, que se refieren únicamente a cuestiones procedimentales para la integración de los Estados al tratado.

Es importante anotar frente al artículo anterior, que el hecho de que las organizaciones internacionales intergubernamentales puedan realizar actividades espaciales no implica que éstas sean internacionalmente responsables de los daños producidos por un objeto espacial o que puedan considerarse como propietarias de un objeto espacial. Esto se debe a que éstas no pueden ostentar la calidad de "Estado de regis- 
tro", pues tampoco pueden considerarse como "Estados de lanzamiento".

Para entender cómo participan las organizaciones intergubernamentales internacionales en el ámbito espacial hay que recordar la definición de "Estado de lanzamiento", específicamente en lo que se refiere al criterio del "Estado que promueva el lanzamiento". Es este criterio el que permite que, independientemente de la forma como se conciba la nacionalidad de estas organizaciones (ya sea según las normas del país donde se creó la organización o el lugar donde ésta tiene su centro administrativo), siempre haya un Estado responsable por las actuaciones de la organización, lo anterior sin perjuicio de los acuerdos internos sobre responsabilidad entre el Estado y la organización.

Siguiendo la línea del Convenio, los artículos que se analizarán a continuación son principalmente artículos que tratan sobre el procedimiento para la ratificación y, entre otros, aspectos formales que son necesarios para la aplicación efectiva del mismo; aun cuando no hablan específicamente sobre el registro de objetos espaciales, es igualmente importante su mención en este escrito, ya sea para que el lector tenga conocimiento básico del proceso o porque de su formalismo puede extraerse también el modus operandi para estos casos, lo cual puede reflejar bajo qué principios opera.

El artículo VIII se refiere al proceso de firma y ratificación del Tratado. Establece Específicamente que el Tratado esta abierto a la firma en la sede de las Naciones Unidas en Nueva York y que aun cuando haya Estados que no lo ratifiquen, previo a la entrada en vigor del mismo, podrán firmar su vinculación al Tratado después. También dicta que luego de la firma, el Tratado queda sujeto a la ratificación del mismo por parte de aquellos Estados que lo firmaron y que los instrumentos de ratificación y adhesión serán entregados al Secretario, caso en el cual, cuando el quinto instrumento sea depositado por los Estados, se entenderá que el tratado entra en vigor para aquellos. No obstante, para aquellos que depositen sus instrumentos luego de esa fecha se entenderá que para efectos de ese Estado en específico, entra en vigor cuando depositó el total de los instrumentos. El Secretario notificará a cada uno de las cuestiones pertinentes como las fechas específicas de ratificación y adhesión.

Posteriormente, el artículo IX describe una situación importante y es la posibilidad que tienen los Estados Parte en el convenio de proponer cambios o ajustes al mismo. Para que esto se pueda efectuar es necesario que la mayoría de los Estados Parte acepten el cambio, situación en la cual se entenderá para todos los Estados que el tratado entra en vigor en ese momento. Sin embargo, los Estados minoritarios, que no lo aceptaron, pueden hacerlo más adelante y se entiende que frente a ellos, la enmienda entra en vigor cuando ellos la acepten. En este punto se puede reflejar como se integra el factor de flexibilidad en el Convenio, pues se entiende que la tecnología es una cuestión que avanza de forma rápida y que desde el momento de la creación del tratado es necesario prever que éste necesite reformase para abracar y adaptarse a las situaciones futuras y distintas que puedan surgir. 
También es claro que tales cambios están sometidos a un régimen básico de votación y que aun cuando se necesite una mayoría para que se realice el cambio, nunca estarán obligados a éste aquellos Estados que votaron en contra, entendiendo por esto que se respeta también si los Estados solo quieren acogerse a lo que aceptaron en principio. Si esto no fuere así no habría seguridad jurídica, puesto que aquellos que aceptaran el primero, estarían obligados a aceptar a priori cosas que no sabrían desde antes, lo cual, podría cambiar su decisión de firmar o no el Tratado.

Adicionalmente, el artículo $\mathrm{X}$ trae una orden de revisión obligatoria del convenio, diez años después de su entrada en vigor, todo con miras a revisar y reformar, como se explicó arriba, aquellas cuestiones que hagan falta o que hayan caído en desuso por el cambio tecnológico. Como ya se vio, es importante el reconocimiento que se hace de antemano de que el convenio puede necesitar cambios futuros. Es esto tan claro que incluso permite una petición para su revisión, cinco años después de su entrada en vigor, si un tercio de los Estados Parte lo requieren.

El Tratado permite además, en el artículo XII, el retiro por parte de los Estados. Para que esto pueda ocurrir es necesario que haya transcurrido un año de la entrada en vigor del tratado y, además, luego de su solicitud ante el Secretario General, ésta se hará efectiva únicamente un año después.

Finalmente, el artículo XII explica que aquellos documentos o tratados del mismo escritos en distintos idiomas al original, son igualmente auténticos y así vinculantes en la medida en que el Secretario lo haya certificado.

\section{Estudio Resoluciones Conexas al Tratado}

Como ya se mencionó, las resoluciones acerca del tema de registro de objetos espaciales son importantes debido a que complementan, armonizan o actualizan el Tratado, por lo que entender esta regulación sin las resoluciones resultaría incompleto e inexacto.

La primera resolución a la que se hará referencia es anterior al Convenio. Esta es la resolución 1721, del 20 de diciembre de 1961. En esta hay un primer avance frente al tema del registro, pues se hace un Ilamado a los Estados de lanzamiento para que presenten lo antes posible al Secretario General, la información pertinente frente a los lanzamientos que se realicen para llevar un registro de las mismas. Se puede notar aquí que de una u otra forma este llamado se ve reflejado en el Tratado, ya que la finalidad principal de éste es registrar todos los objetos que sean lanzados al espacio ultraterrestre.

Así mismo, al igual que en el Tratado bajo estudio, la resolución pide al Secretario General que mantenga la información del registro pública, lo cual muestra que desde un inicio se quería materializar el principio de reciprocidad del que ya se habló.

La importancia de esta resolución radica entonces en mostrar que ya desde 1961, o incluso 
desde antes se estaba materializando la necesidad que tenían los Estados y la ONU de crear un mecanismo que recopilara todas aquellas actividades realizadas tanto por Estados como por organizaciones intergubernamentales internacionales. Se puede concluir de la resolución que fue el primer acercamiento a la constitución del registro que se conoce hoy. A pesar de las buenas intenciones que se pretendían al principio con la promulgación de la resolución, actualmente esta presenta un problema para el Tratado bajo examen, el cual se explicará más adelante.

La resolución 41/66, del 3 de diciembre de 1986, es relevante ya que atiende al Ilamado del artículo $\mathrm{X}$ del Convenio sobre el registro de objetos lanzados al espacio ultraterrestre, en donde, como se expuso anteriormente, se invita a los Estados a que diez años después de su entrada en vigor, revisen el tratado para que se realicen los ajustes necesarios para actualizarlo frente a los cambios tecnológicos. Así pues, surge la resolución mencionada, en donde se establece lo siguiente: 1.) que el convenio sigue siendo importante y necesario debido al aumento de la actividad en el espacio ultraterrestre. En razón de esto, 2) hace un llamado a los Estados que no han ratificado aun el tratado y, de forma específica, a aquellos que realizan actividades en el espacio ultraterrestre para que lo hagan con urgencia. 3) del mismo modo, invita a las organizaciones gubernamentales internacionales que realizan actividades espaciales, en virtud del artículo VII, a que se adhieran al Tratado.

Es importante mencionar aquí que, para la fecha de esta resolución, treinta y cinco Estados habían ratificado el tratado y que cinco lo habían firmado (Asamblea de las Naciones Unidas, 1986, Cuestión del nuevo examen del Convenio sobre registro de objetos lanzados al espacio ultraterrestre), Lo cual, en mi opinión y teniendo en cuenta la época es un número que demuestra el compromiso por parte de los países que realizaban actividades espaciales. No obstante, se debe aceptar que la aplicación efectiva de un tratado de este tipo, donde se quiere llevar un registro de los objetos lanzados al espacio ultraterrestre, (y teniendo en cuenta las implicaciones mencionadas que esto trae), no va a poder ser totalmente efectiva si no es acogida por un número importante de Estados.

La resolución 62/101, del 10 de enero de 2008, al igual que en la anterior, invita a los Estados y organizaciones intergubernamentales internacionales que no hagan parte aun del Tratado, a que se vinculen lo más pronto, teniendo en cuenta que entre más Estados y organizaciones pertenezcan al mismo, mayor es la utilidad que el Convenio provee. Esta situación demuestra que aún hay un gran número de Estados y organizaciones que, a pesar del tiempo que lleva en vigencia el Tratado y a pesar de la necesidad que hay en que todos lo ratifiquen, aún no lo han hecho.

Además de esto, la resolución trae un avance y es aquí donde se encuentra su mayor importancia. Ésta tiene como objetivo pedir que se haga un estudio para darle uniformidad a la información que se envía o se imprime en el registro, por lo que recomienda unas pautas a los países para que tengan claro cuál es el tipo de informa- 
ción que se requiere de forma primordial para que eventualmente se realice el cambio formal. En pocas palabras, es posible atreverse a decir que se busca especificar más los requisitos que trae el artículo IV del presente Tratado. Estas recomendaciones son las siguientes:

i) La designación internacional del Comité de Investigaciones Espaciales, cuando resulte procedente;

ii) La hora universal coordinada como referencia cronológica de la fecha de lanzamiento;

iii) Kilómetros, minutos y grados como unidades tipo de los parámetros orbitales básicos;

iv) Toda información útil relativa a la función del objeto espacial, además de la correspondiente a su función general que debe presentarse conforme al Convenio sobre el registro. (Asamblea General de las Naciones Unidas, 2008, Recomendaciones para mejorar la práctica de los Estados y las organizaciones intergubernamentales internacionales en cuanto al registro de objetos espaciales, Art. 2(a)).

Se pide también en la resolución que dentro de lo posible, se incluya en el registro la información que se refiere en general a los cambios que puedan acaecer en la operación del objeto y las fechas de "salida" de la órbita de los mismos:

i) La ubicación en la órbita geoestacionaria, cuando proceda;

ii) Toda modificación de la situación de las operaciones (entre otras cosas, si un objeto espacial ha dejado de estar en funcionamiento); iii) La fecha aproximada de desintegración o reingreso, en caso de que los Estados puedan verificar esa información;

iv) La fecha y las condiciones físicas de traslado de un objeto espacial a una órbita de eliminación;

v) Enlaces a sitios web con información oficial sobre objetos espaciales. (Asamblea General de las Naciones Unidas, 2008, Art. 2 (b)).

Es menester hacer énfasis en la importancia específica que tienen los numerales I, III y IV para la órbita geoestacionaria. Teniendo claro que esta órbita es un recurso limitado (puesto que el fenómeno que ahí se presenta es único y solo consta de $360^{\circ}$ ) es de gran importancia saber cuándo se posiciona un objeto especial ahí y, a priori, tener conocimiento de cuándo va a quedar libre aquella posición para que se pueda ocupar de nuevo por otro objeto.

Además, en aras de cubrir la totalidad de los objetos enviados al espacio ultraterrestre en el registro, pide la Resolución 62/101 que se busque una solución en cuanto a la situación de las organizaciones gubernamentales internacionales compuestas por Estados que realizan actividades espaciales que aún no se han adherido al tratado y conmina a ponerse de acuerdo para solucionar esta situación.

Igualmente, busca que aquellos lanzamientos, en los cuales los Estados u organizaciones de lanzamiento no se hayan puesto de acuerdo en cuanto a quién va a inscribir el lanzamiento en su registro, el Estado desde cuyo territorio se 
haya lanzado se ponga en contacto para decidir esta cuestión. Asimismo especifica que en los lanzamientos que se den de forma conjunta entre Estados, los involucrados registren de forma separada tal situación.

Finalmente, frente a las transferencias de control de un objeto espacial que se realice de un Estado a otro (o entre organizaciones), la resolución en cuestión pide que se suministre la siguiente información: 1.) La fecha en que se transfiere el control del objeto; 2.) La designación del nuevo propietario o entidad explotadora; 3.) Cualquier cambio de la posición orbital que se vaya a llevar a cabo y 4.) Cualquier cambio que se le dé a la función principal del objeto espacial.

Con la anterior exposición se pueden visualizar las nuevas necesidades que han surgido frente a la especificación de la información que se requiere en el registro, así como necesidades anteriores que aún no se han podido satisfacer.

\section{BENEFICIOS EN LA APLICACIÓN DEL TRATADO}

A partir del anterior estudio y análisis es posible extraer varias ventajas que otorga el tratado bajo examen.

\section{A. Jurisdicción y Control}

La ventaja o efecto más importante del presente Tratado es que el registro que impone declara a la comunidad internacional que la propiedad de cierto objeto esta en cabeza de algún Estado y que aun cuando la posición orbital o cuerpo celeste donde se encuentre no puede ser sujeto de apropiación, el objeto lanzado sí. Esto quiere decir que en un lugar donde nada es apropiable, los objetos lanzados al espacio son los únicos que ya están sujetos a la propiedad de algún Estado cuando alcanzan su posición en el espacio ultraterrestre y mantienen esta característica siempre. De este modo, la relación entre el Estado de lanzamiento y el objeto lanzado al espacio otorga derechos al Estado de Lanzamiento, como es la obligación de que el objeto se le restituya a él si se ha extraviado y obligaciones, como la de responder por cualquier daño que el objeto espacial cause.

\section{B. Aplicación de los Principios que deben regir las actividades espaciales}

Además de lo anterior, es debido reconocer que el Tratado no desconoce los principios generales de la actividad espacial sino que, por el contrario, las razones o motivos por los que se realizó el convenio se ajustan y además propenden por la aplicación de dichos principios.

En primera instancia, asegura la aplicación del principio que busca mantener el uso del espacio de manera pacífica, pues si la información que se inscribe es además corroborada, se puede reducir la posibilidad de que los objetos enviados cumplan funciones ilegales o que contravengan los principios.

Así mismo, teniendo en cuenta la resolución 80 del 2000 de la UIT, que busca dar aplicación 
a los tratados de Nairobi y de Málaga-Torremolinos, si se pretende dar beneficios a los países en desarrollo frente a posiciones orbitales, la propiedad juega un papel importante, pues permite también dar información a la UIT de cuántas posiciones tiene un país desarrollado, un país en vía de desarrollo y cuáles no han obtenido ninguna, para que de esta forma no se aplique el principio de "primer llegado, primer servido" sin discreción sino, teniendo en cuenta las situaciones en que se encuentran los países que buscan acceder a una posición. Así, si dos países pretenden acceder a una posición orbital en un mismo momento y hay uno que es subdesarrollado y nunca ha accedido a una posición y, el otro sí, con este conocimiento que deviene del registro, la UIT puede decidir con mayor objetividad quién merece la nueva posición (a quien eventualmente se le podría adjudicar, siempre que se hablara de la existencia de un régimen jurídico especial frente al tema). De igual manera, la información que contiene el registro permite saber cuándo habrán de desocuparse posiciones orbitales en la órbita de los satélites geoestacionarios, lo que implica que los países que necesiten acceder puedan solicitarla. Todo lo anterior se da en relación con el principio de cooperación internacional del cual se derivan, además, los principios del uso equitativo y eficaz de la órbita de los satélites geoestacionarios.

Además, por medio del registro el principio de reciprocidad encuentra un apoyo, pues teniendo una información clara y certera en manos de la ONU, esta puede ser publicada fácilmente para que todos los Estados tengan conocimiento de qué actividades están llevando a cabo los otros, sin que necesariamente tengan que contactarse con ellos directamente o, en su defecto, estar en su territorio. Esto también permitiría que la actividad espacial sea transparente.

\section{Aplicación efectiva de otros tratados}

En segundo lugar, el convenio ayuda a que la aplicación de otros tratados sea eficaz y efectiva, por ejemplo, haciendo referencia al Tratado de responsabilidad por daños ocasionados por objetos espaciales, el Convenio facilita a los Estados víctimas de un daño ocasionado por algún objeto espacial, la identificación del Estado propietario del objeto y como consecuencia de esto, hace más efectivo y rápido el pago de aquellos daños.

Del mismo modo, la información de un objeto contendida en el registro puede ayudar a identificar si el daño se ocasionó con culpa o dolo del Estado propietario, lo cual es importante si se tiene en cuenta que la responsabilidad en el espacio ultraterrestre es subjetiva debido a que el registro posee información detallada sobre la posición orbital, periodo nodal, inclinación, velocidad, etc., caso en el cual, un experto puede valerse de dichos datos para indicar si el daño fue fruto de la culpa del Estado o del dolo.

Así mismo, toda la información anterior más aquella referente al lanzamiento, (fecha, hora, ventana de lanzamiento, distancia de la tierra a la posición, etc.), puede ayudar a identificar si un daño que se produce en tierra debe ser atribuible objetivamente al Estado de Lanzamiento 
o si existe la posibilidad de aplicar la única causal de exclusión que es el hecho exclusivo de la víctima.

El registro de los objetos espaciales puede aportar también en la aplicación más efectiva del Acuerdo sobre el salvamento y devolución de astronautas y la restitución de objetos lanzados al espacio ultraterrestre, pues como se expuso en el párrafo anterior, la información contenida en el registro es pública y cierta y así se puede identificar con facilidad a los objetos pertenecientes a determinado Estado, y los astronautas enviados por el mismo, para que se pueda dar una restitución y salvamento más efectivos si llagare a necesitarse.

Finalmente, este tratado puede ayudar con la aplicación de la resolución de mitigación de desechos espaciales debido a que en los registros esta estipulada la fecha de salida de órbita de los objetos espaciales, se puede llevar un inventario de cuántos y cuándo los objetos espaciales caerán en desuso y en qué orbitas (MEO, LEO, GEO), para que los mecanismos de mitigación de desechos puedan ser aplicados de forma más efectiva.

\section{PROBLEMÁTICAS EN LA APLICACIÓN DEL TRATADO}

También es importante reconocer que el tratado tiene ciertos problemas en cuanto a su aplicación se refiere.

\section{A. Existencia de otros registros}

En primer lugar esta el hecho de que exista una resolución anterior, específicamente la 1721 B, de 20 de Diciembre de 1961, la cual, como ya se expuso anteriormente, hace un llamado a los Estados para que de la manera más rápida posible envíen información al Secretario General de la ONU y le exige a éste último mantener un registro público. El problema con dicha resolución es que aún se mantiene vigente y aún existen Estados que utilizan lo dispuesto por esta resolución para hacer el registro y no lo que establece el Convenio sobre registro de objetos lanzados al espacio ultraterrestre. Lo anterior implica que existan dos registros paralelos con distintas exigencias para su conformación y distintos derechos u obligaciones otrogados. Esto supone un problema en la homogeneidad que se requiere para que el registro pueda propiciar las ventajas expuestas con anterioridad. Se ha demostrado además que los Estados que hacen uso del registro regulado por la Resolución 1721 B, de 20 de Diciembre de 1961, son aquellos que aún no hacen parte del tratado en cuestión.

Adicional a estos dos registros, el que establece la resolución expuesta y el Tratado bajo examen, existen tres registros adicionales que tratan materias mucho más específicas. Uno de ellos es el Manual de Cohetes y Satélites de 1962, el cual permite el intercambio de información importante para organizaciones científicas y estaciones de observación que puede resultar beneficiosa para los avances científicos. Otro registro es aquel que lleva la UIT sobre las frecuencias 
asignadas a redes de satélites, en donde estaciones espaciales y sus equivalentes en la tierra son registradas. Finalmente, existe un registro que se refiere a cuestiones de seguridad en los objetos espaciales que se dio en la "Convention on International Interests in Mobile Equipment". Aun cuando estos registros no busquen contener la misma información que pretende recoger el registro del Convenio sobre objetos lanzados al espacio ultraterrestre, la necesidad de mantener registradas diversas cuestiones sobre la actividad espacial es evidente. Por lo tanto, no esta demás pensar que un registro unificado sobre los aspectos importantes referentes a objetos lanzados al espacio ultraterrestre beneficiaría aún más a los Estados y organizaciones espaciales en sus actividades.

\section{B. Ratificación del Tratado}

En concordancia con lo anterior, otro de los problemas que enfrenta el Tratado es que no todos los Estados u organización internacionales intergubernamentales lo han ratificado, lo que implica que no se pueden predicar todas aquellas ventajas o características que el Tratado otorga a los Estados que realizan actividades espaciales, como los son la identificación, la información sobre su posición, la información sobre la fecha en que caerá en desuso y la efectiva aplicación de los demás Tratados como el de responsabilidad por daños, el salvamento y devolución de astronautas y restitución de objetos, entre otros. Por lo tanto, que solo unos cuantos Estados estén sometidos al Tratado hace que su aplicación no pueda darse de la forma más efectiva ya que para ello es necesario que existan unas reglas comunes a todos que permita el desarrollo óptimo del mismo.

\section{CONCLUSIÓN}

Se puede afirmar, entre otras cosas, que el Tratado sobre registro de objetos lanzados al espacio ultraterrestre es un tratado muy importante para las actividades que se realizan en el espacio ultraterrestre porque permite otorgar a un Estado la propiedad de objetos que van a ser lanzados al espacio y, en ese sentido, sobre él recaerá la jurisdicción y el control del objeto cuando llegue al espacio ultraterrestre, lugar en el cual ningún objeto es apropiable. Así mismo, la obligación de registrar los objetos espaciales a nombre de un Estado otorga a éste último obligaciones y derechos que no serían posibles si no existiese la propiedad sobre los objetos. No obstante, es importante tener en cuenta que nada de lo anterior puede aplicarse de forma completamente efectiva si la mayoría de Estados que realizan actividades espaciales no se acogen al mismo y si existe otra variedad de registros que se presten para confusiones por las distintas obligaciones y derechos que otorgan ${ }^{3}$.

\section{Bibliografía}

Asamblea General de las Naciones Unidas. (1967). Tratado sobre los principios que deben regir las actividades de los Estados en la exploración y utilización del espacio ultrate-

3 Este Tratado, ha sido ratificado por el Congreso Colombiano, mediante la Ley 1569 de 2012, que posteriormente deberá pasar al control Constitucional de la Corte. 
rrestre, incluso la Luna y otros cuerpos celestes. Aprobado por la Resolución 2222 (XXI), de 19 de diciembre de 1966. Recuperado de http://www.oosa.unvienna.org/pdf/publications/STSPACE11S.pdf

---. (1967). Acuerdo sobre el salvamento y la devolución de astronautas y la restitución de objetos lanzados al espacio ultraterrestre. Aprobado por la resolución 2345 (XXII), de 19 de diciembre de 1967. Recuperado de http:// www.oosa.unvienna.org/pdf/publications/ STSPACE11S.pdf

---. (1969). Convención de Viena sobre el Derecho de los Tratados. Recuperado de http:// www.derechos.org/nizkor/ley/viena.html

---. (1970). Declaración relativa a los principios de derecho internacional referentes a las relaciones de amistad y a la cooperación entre los estados de conformidad con la Carta de las Naciones Unidas. Aprobada por la Resolución 2625 (XXV), de 24 de octubre de 1970. Recuperado de http://www.aloj.us.es/ eulalia/derecho\%20internacional/materiales\%20dpto/resolucion2625.htm

---. (1971). Convenio sobre la responsabilidad internacional por daños causados por objetos espaciales. Aprobada por la Resolución 2777 (XXVI), de 29 de noviembre de 1971. Recuperado de http://daccess-dds-ny.un.org/ doc/RESOLUTION/GEN/NRO/332/55/IMG/ NR033255.pdf?OpenElement ---. (1974). Convenio sobre el registro de objetos lanzados al espacio ultraterrestre. Aprobado por la Resolución 3235 (XXIX), de 12 de noviembre de 1974. Recuperado de http:// www.oosa.unvienna.org/pdf/publications/ ST_SPACE_51S.pdf

---. (1986). Cuestión del nuevo examen del Convenio sobre registro de objetos lanzados al espacio ultraterrestre. A/RES 41/66 de 3 de Diciembre de 1986. Recuperado de http:// www.un.org/es/comun/docs/?symbol=A/ RES/41/66

--. (2006). Directrices para la reducción de desechos espaciales elaboradas

por la Subcomisión de Asuntos Científicos y Técnicos de la Comisión sobre la Utilización del Espacio Ultraterrestre con Fines Pacíficos. Informe sobre la marcha de los trabajos presentado por el Presidente del Grupo de Trabajo del Grupo de Trabajo sobre desechos espaciales A/AC.105/C.1/L.284 de 28 de febrero de 2006. Recuperado de http://www. oosa.unvienna.org/pdf/limited/c1/AC105_ C1_L284S.pdf

---. (2008). Recomendaciones para mejorar la práctica de los Estados y las organizaciones intergubernamentales internacionales en cuanto al registro de objetos espaciales $A$ / RES 62/101 de 10 de enero de 2008. Recuperado de http://www.oosa.unvienna.org/ pdf/gares/ARES_62_101S.pdf

Benko, M., \& Schrogl. K. (Eds.). (2005). Essential Air and Space Law. 
The Registro de Objetos Espaciales. (s.f.). Recupe-

Netherlands: Eleven International Publishing.

Duque, E., Y Pérez, A. (2012). Objetos Espaciarado de http://www.enciclopedia-juridica. biz14.com/d/registro-de-objetos-espaciales/ les. Derecho del Espacio Ultraterrestre. Bogotá, Colombia: Universidad de los Andes. registro-de-objetos-espaciales.htm

Ternera Barrios, F. (2011). Bienes. Bogotá, CoIombia: Editorial Universidad del Rosario. Bo-

Ferrer, M. (1976). Derecho Espacial. Buenos Aires, Argentina: Editorial Plus Ultra. gotá, 2011. 